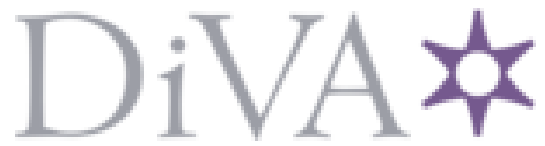

http://www.diva-portal.org

\title{
Postprint
}

This is the accepted version of a paper published in . This paper has been peer-reviewed but does not include the final publisher proof-corrections or journal pagination.

Citation for the original published paper (version of record):

Zheng, J., Jongcharoenkamol, J., Peters, B B., Guhl, J., Ponra, S. et al. (2019)

Iridium-catalysed enantioselective formal deoxygenation of racemic alcohols via asymmetric hydrogenation

Nature Catalysis, 2(12): 1093-1100

https://doi.org/10.1038/s41929-019-0375-7

Access to the published version may require subscription.

N.B. When citing this work, cite the original published paper.

Permanent link to this version:

http://urn.kb.se/resolve?urn=urn:nbn:se:su:diva-177599 


\section{Iridium-Catalysed Enantioselective Formal Deoxygenation of Racemic Alcohols via Asymmetric Hydrogenation}

Jia Zheng ${ }^{1}$, Jira Jongcharoenkamol ${ }^{1 \ddagger}$, Bram B. C. Peters ${ }^{1}$, Jasper Guhl ${ }^{1}$, Sudipta Ponra ${ }^{1}$, Mårten S. G. Ahlquist ${ }^{2}$, Pher G. Andersson ${ }^{1,3 *}$

${ }^{1}$ Department of Organic Chemistry, Stockholm University, Svante Arrhenius väg 16C, SE-10691 Stockholm, Sweden.

${ }^{2}$ Department of Theoretical Chemistry \& Biology, School of Engineering Sciences in Chemistry Biotechnology and Health, KTH Royal Institute of Technology, SE-10691 Stockholm, Sweden.

${ }^{3}$ School of Chemistry and Physics, University of Kwazulu-Natal, Private Bag X54001, Durban, 4000, South Africa.

*e-mail: $\underline{\text { Pher.Andersson@su.se }}$

Abstract: Asymmetric hydrogenation of alkenes is today one of the most powerful tools for the preparation of optically active compounds. However, in order to have high enantioselectivity, the starting olefin in most cases needs to be isomerically pure in either cis or trans form. Generally, most olefination protocols provide olefins as isomeric mixtures that are difficult to separate and in many cases also generate lots of waste. In contrast, the synthesis of racemic alcohols is straightforward, easier to purify and is very atom efficient. Here, we describe a strategy that enables rapid access to chiral alkanes via enantioconvergent formal deoxygenation of racemic alcohols. Mechanistic studies indicate an Ir-mediated elimination of water and subsequent in-situ hydrogenation. This approach allows rapid and efficient assembly of chiral intermediates and is exemplified in the total synthesis of antidepressant sertraline and $\sigma_{2}$ receptor PB 28.

Asymmetric hydrogenation of olefins has emerged as one of the most efficient and atom-economic strategies to install steric centers to prochiral compounds, particularly in industry. ${ }^{1-7}$ In general terms, geometrically pure alkenes are required to produce products in high enantiomeric excess due to the enantiodivergence phenomenon. ${ }^{8-13}$ The synthesis of olefins normally produce isomeric olefin mixtures which require difficult chromatographic separation. In addition, the classic olefination processes also generate a considerable amount of byproducts, such as phosphine oxide or phosphinate and residues from leaving groups and bases (Fig. 1a). ${ }^{14-16}$ In contrast, tertiary alcohols can be simply prepared by addition to ketones with very little by-product formation and are easily purified. The combination of alcohol preparation and deoxygenation process could transform ketones into chiral alkanes with water as the only byproduct, providing an efficient and atom-economic approach. Therefore, asymmetric deoxygenation of racemic alcohols could be an ideal alternative to produce chiral alkanes. Over the past few decades, inspired by the Barton-McCombie reaction, numerous methods for deoxygenation have been developed by using transition metal, Lewis- or Brønsted acid catalysts. ${ }^{17-19}$ Recently, strategies like mono-deoxygenation of diols, ${ }^{20-24}$ sequential dehydrogenation/Wolff-Kishner reduction, 25-27 and directing group-assisted site-selective deoxygenation ${ }^{28}$ have been utilized to tackle the regioselective deoxygenation process. However, the construction of enantioenriched tertiary carbons via deoxygenation of racemic alcohols remains challenging. The only related strategy recently reported by Carreira's group was the transition-metal catalysed allenylic substitution, using an allene group for the coordination-induced enantiocontrol. ${ }^{29}$ Despite the above advance in this concept, the development of enantioselective deoxygenation of unfunctionalized alcohols is still challenging and in great demand. 
We herein report a strategy for enantioselective deoxygenation of racemic alcohols via N,P-iridium catalysis, which allows the facile construction of chiral alkanes with good to excellent e.e.s (Fig. 1, b). This protocol opens up a direct route to create stereogenic centers in a very efficient and atomeconomic approach.

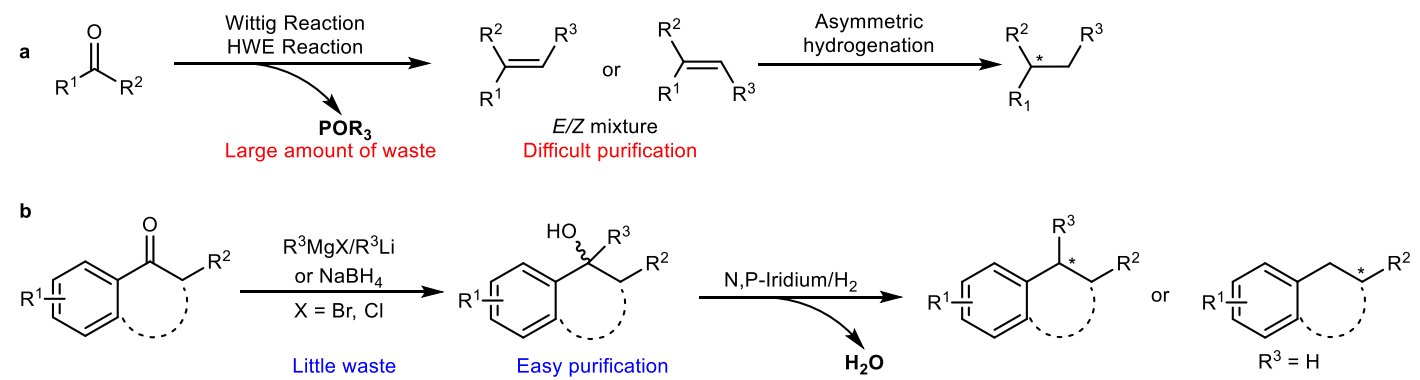

Fig. 1. Different preparative routes from ketones to chiral alkanes. a, Classic olefination followed by asymmetric hydrogenation. b, This work: alcohol preparation followed by enantioselective deoxygenation.

\section{Results}

Initial studies. In our previous studies, the acidic $\operatorname{Ir}(\mathrm{V})$-hydride species showed possibilities to cleave alcoholic $\mathrm{C}-\mathrm{O}$ bonds. ${ }^{30-31}$ Recently, we accidentally observed that the $\alpha, \beta$-unsaturated $t$-butyl ester was quantatively converted into the chiral carboxylic acid upon attempted asymmetric hydrogenation (Fig. 2a). ${ }^{32}$ Even though it was not isolated, we suspected that isobutane was formed as a byproduct by either hydrogenolysis or elimination followed by hydrogenation. To confirm this hypothesis, the $t$-butyl group was replaced by a dimethylbenzyl group. Subjecting 2-phenylpropan-2-acetate to typical hydrogenation conditions using N,P-iridium catalyst $\mathbf{i}$, resulted in the formation of cumene which was confirmed by ${ }^{1}$ H-NMR (Fig. 2b). Furthermore, the corresponding alcohol, 2-phenylpropan-2-ol, was fully converted into cumene under the same reaction conditions (Fig. 2c). Inspired by the above results, racemic substrate 1a was selected to investigate the possibility of an asymmetric deoxygenation in our catalytic system. Gratifyingly, N,P-iridium catalyst $\mathbf{i}$ gave full conversion of racemic alcohol 1a to $2 \mathbf{a}$ with $81 \%$ e.e. in DCM under 20 bar of $\mathrm{H}_{2}$ (Fig. 2d). We also found that a number of related catalysts such as $\left[\operatorname{Ir}(\mathrm{Py})\left(\mathrm{PCy}_{3}\right)\right] \mathrm{BAr} \mathrm{F}_{\mathrm{F}}$, Crabtree's catalyst and Wilkinson's catalyst did not trigger the deoxygenation under the same conditions. Moreover, when the $\operatorname{Ir}(\mathrm{COD}) \mathrm{Cl}$ dimer was used as catalyst without any ligand, no deoxygenation took place and only formation of Ir-based nanoparticles was observed. 


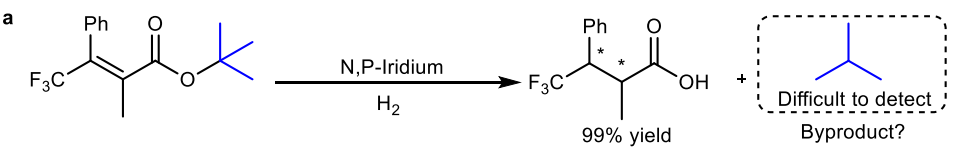

C-O cleavage via Hydrogenolysis or elimination of carbocation?

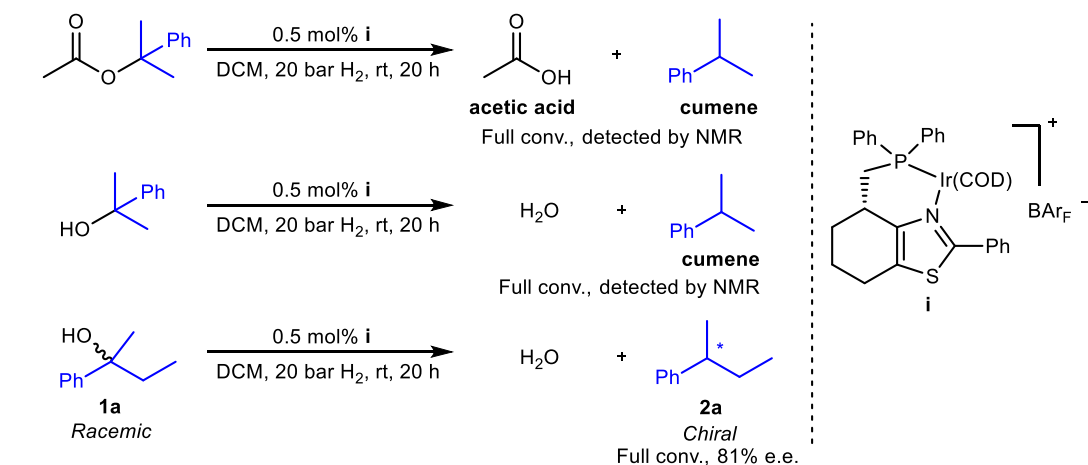

Fig. 2. Initial studies of Ir-catalysed deoxygenation. a, Observed $\mathrm{C}-\mathrm{O}$ bond cleavage in previous study. b, Initial attempt of acetate deoxygenation. c, Initial attempt of alcohol deoxygenation. d, Initial attempt of asymmetric deoxygenation.

Optimization studies. Next, an optimization was carried out by using 1a as the model substrate. In the solvent screening, $\mathrm{PhCF}_{3}$ gave $85 \%$ e.e. which is higher than that obtained in $\mathrm{DCM}$, benzene and toluene (Table 1, entries 1-4). The reaction also proceeded smoothly in EtOAc but gave low enantioselectivity (48\% e.e., Table 1, entry 5). As expected, solvents like THF and $i$-PrOH inhibited the transformation since the coordination between the substrate and catalyst was blocked (Table 1, entries 6-7). Therefore, the following catalyst screening was run in $\mathrm{PhCF}_{3}$. Catalysts with different backbones were selected (catalysts $\mathbf{i}-\mathbf{v}$ ) from our catalyst library for the initial catalyst optimization. It is worth noting that the selected catalysts had shown excellent enantioselectivity in the hydrogenation of the unfunctionalized trisubstituted $E$-olefin (probable intermediate) since we speculated an olefination process was involved in this deoxygenation. To our surprise, these catalysts afforded different e.e.s in the model reaction, which indicated that the elimination might generate isomeric olefin mixtures instead of pure E-olefin. To our delight, we found that linear alcohol 1a fitted well to the imidazole catalyst ii, giving high enantioselectivity (91\% e.e., Table 1, entry 8). Oxazole and oxazoline catalysts iii and iv afforded only moderate e.e.s while thiazole catalyst $\mathbf{v}$ resulted in $81 \%$ e.e. (Table 1, entries 9-11). To further improve the enantiomeric outcomes, several other catalysts were evaluated (Supplementary Table 1). Unfortunately, varying the nature of the phosphine or heterocyclic moieties did not improve the enantiomeric excess. Therefore, imidazole catalyst ii in $\mathrm{PhCF}_{3}$ was found to be the best combination for the enantioselective deoxygenation of linear alcohols.

Table 1. Selected optimization for linear alcohol

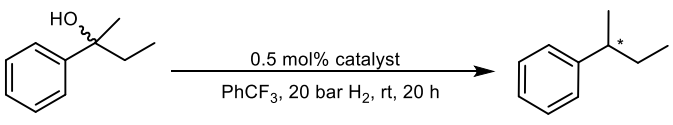

1a

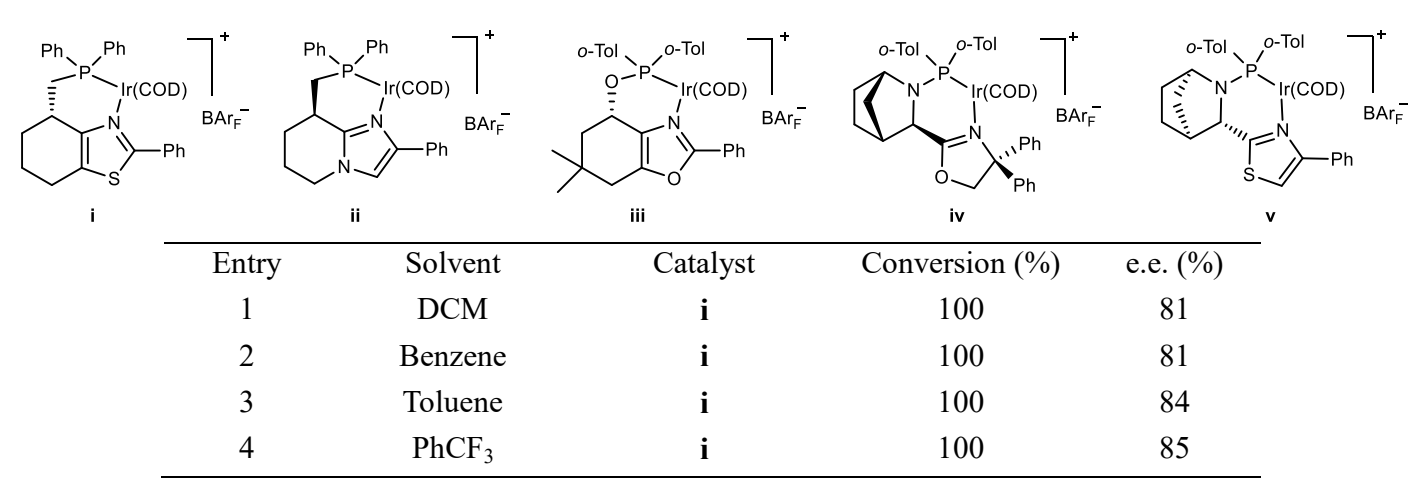




\begin{tabular}{ccccc}
\hline 5 & EtOAc & i & 100 & 48 \\
6 & $\mathrm{THF}$ & $\mathbf{i}$ & 0 & - \\
7 & $i$-PrOH & i & 0 & - \\
$\mathbf{8}$ & $\mathbf{P h C F}_{3}$ & ii & $\mathbf{1 0 0}$ & $\mathbf{9 1}$ \\
9 & $\mathrm{PhCF}_{3}$ & iii & 100 & 55 \\
10 & $\mathrm{PhCF}_{3}$ & iv & 100 & 65 \\
11 & $\mathrm{PhCF}_{3}$ & $\mathbf{v}$ & 100 & 81
\end{tabular}

Reaction conditions: $0.1 \mathrm{mmol}$ substrate, $0.5 \mathrm{~mol} \%$ catalyst, $0.5 \mathrm{~mL} \mathrm{PhCF}$. Conversions were determined by ${ }^{1} \mathrm{H}-$ NMR spectroscopy. Enantiomeric excess determined by GC analysis using a chiral stationary phase.

Scope of enantioselective deoxygenation. Having optimized the conditions, we investigated the substrate scope of linear tertiary alcohols. As shown in Fig. 3, an electron-withdrawing group led to lower reactivity, and required $1 \mathrm{~mol} \%$ catalyst loading to reach full conversion to yield $\mathbf{2 b}$ with $88 \%$ e.e. Substrates with electron-donating groups on the para- and meta-position (1c-1e) gave excellent isolated yields (88-95\%) and enantioselectivities (87-91\% e.e.s). The 1,1'-biphenyl and 2-napthyl substrates were deoxygenated to generate $\mathbf{2 f}$ and $\mathbf{2} \mathbf{g}$ with $94 \%$ e.e. where the yield of $\mathbf{2} \mathbf{g}$ was slightly lower. Different alkyl chains slightly influenced the enantioselectivity of this transformation, with similar reactivity (92-98\% yields). By increasing the steric hindrance, the enantiomeric excess values varied from $83 \%$ to $87 \%$ when the side chain was substituted by benzyl, $n$-propyl and $i$-propyl groups (2h-j). With benzyl groups adjacent to the quaternary carbon, compounds $\mathbf{1 k}$ and $\mathbf{1 1}$ provided $93 \%$ and $96 \%$ yield with $86 \%$ and $88 \%$ e.e., respectively. When the phenyl adjacent to the deoxygenated center was replaced by $t$-butyl $(\mathbf{1 m})$, only a complicated mixture was obtained. Interestingly, the bidentate diol, compound 1n gave no conversion under the standard conditions, which might be due to chelation between Ir and the hydroxyl groups resulting in a deactivation of the catalyst.

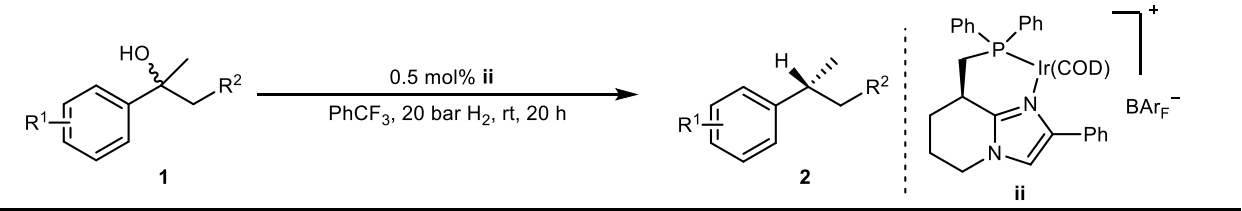

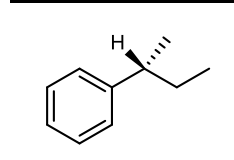

$2 \mathrm{a}$
$87 \%$ yield, $91 \%$ e.e.
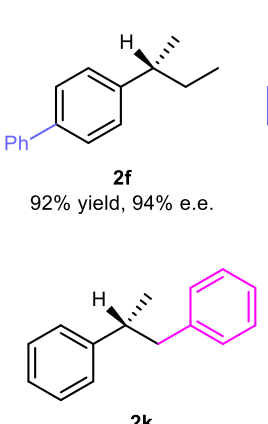

$93 \%$ yield, $86 \%$ e.e. ${ }^{\text {a }}$

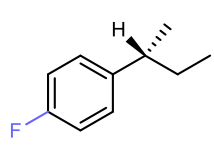

2b 2 b

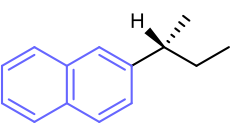

2g

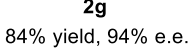

$84 \%$ yield, $94 \%$ e.e.

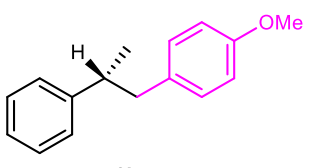

$96 \%$ yield,

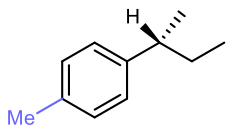

2c

$95 \%$ yield, $93 \%$ e.e.

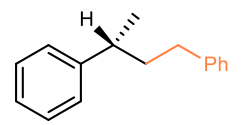

2h

$98 \%$ yield, $85 \%$ e.e.

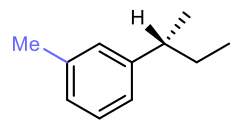

2d
$88 \%$ yield, $89 \%$ e.e

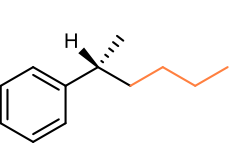

2i

$90 \%$ yield, $87 \%$ e.e

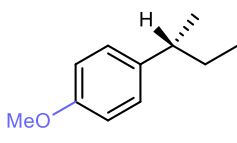

92\% yield, $91 \%$ e.e.
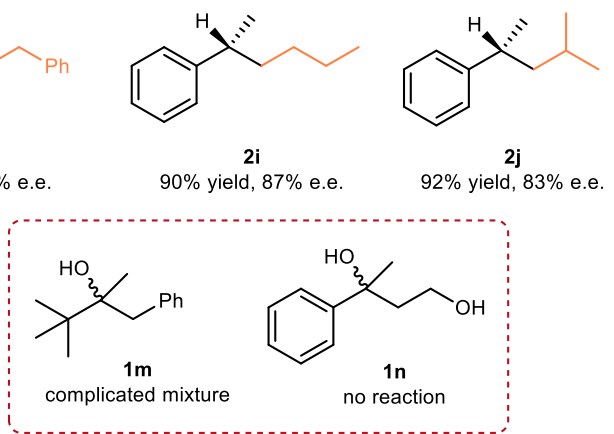

Fig. 3. Substrate scope of linear tertiary alcohols. Reaction conditions: $0.1 \mathrm{mmol}$ substrate, $0.5 \mathrm{~mol} \%$ catalyst, $0.5 \mathrm{~mL} \mathrm{PhCF}_{3}$. Isolated yield. ${ }^{a} 1.0 \mathrm{~mol} \%$ catalyst. Enantiomeric excess determined by GC and SFC analysis using a chiral stationary phase, see Supplementary Table 5 for details.

We then investigated the enantioselective deoxygenation of cyclic tertiary alcohols (Fig. 4a). A catalyst screening (Supplementary Table 2) was required since catalyst ii showed to be less efficient for this class of substrates. Catalysts iii and iv turned out to be best in terms of the selectivity. In addition, similar e.e. values were obtained when hydrogenating cyclic tertiary substrates by both catalysts iii and 
iv (Supplementary Table 3). Increasing the aliphatic chain length from methyl to $n$-butyl gave no obvious influence, producing $\mathbf{4 a - 4 d}$ in high yields with $91 \%$ to $93 \%$ e.e.s. To our delight, $98 \%$ e.e. was observed when a bulkier isopropyl group was installed onto the alcohol carbon (4e). In addition, substrate bearing an alkyl trifluoromethyl group converted smoothly into the desired product with $94 \%$ e.e. (4f). A benzyl substituted alcohol was converted smoothly into $\mathbf{4 g}$ in $92 \%$ yield with $90 \%$ e.e., while the phenyl substituted molecule $\mathbf{4 h}$ was obtained with $99 \%$ e.e.. The removal of the paramethoxyl group on the aromatic ring led to a slight drop of enantioselectivity (4i). Substrates with seven- and five-member rings were examined, affording $\mathbf{4 j}$ and $\mathbf{4 k}$ in high yields with $94 \%$ and $98 \%$ e.e., respectively. Next, the less reactive secondary alcohols were evaluated by using catalyst iv (See Supplementary Table 4 for the catalysts screening), which provided the enantioenriched carbon adjacent to the deoxygenated center. Diastereomeric alcohol mixtures with methyl and benzyl groups proceeded smoothly to afford $\mathbf{6} \mathbf{a}$ and $\mathbf{6 b}$ in high yields with excellent e.e.s. Use of a substrate having a methoxy group on the phenyl ring generated $\mathbf{6 c}$ in $97 \%$ yield with $99 \%$ e.e.. Switching the methyl sidechain to bulkier $i$-butyl group showed no influence on the reactivity or enantioselectivity. The scalability of this Ir-catalysed asymmetric deoxygenation reaction was confirmed by employing $0.88 \mathrm{~g}$ $(4.0 \mathrm{mmol})$ of starting material 3c. The desired product $\mathbf{4 c}$ was obtained in $94 \%$ isolated yield and $93 \%$ e.e.. Both yield and enantioselectivity were retained compared to small scale transformation. To demonstrate the synthetic utility, this strategy was applied to synthesize intermediates of bioactive compounds. Chiral tetrahydronaphthalene $\mathbf{4 l}$ was obtained via the enantioselective deoxygenation in $96 \%$ yield and $98 \%$ e.e., which was a reported intermediate in the synthesis of the antidepressant sertraline (Fig. 4b). ${ }^{33}$ Moreover, racemic alcohol $\mathbf{3 m}$ was fully reduced to afford $\mathbf{4 m}$ in $94 \%$ yield and $88 \%$ e.e.. The following deprotection of the benzyl group led to a chiral alcohol 7 with $88 \%$ e.e., which can be converted into a $\sigma_{2}$ receptor ligand PB 28 (Fig. 4c). ${ }^{34}$ 


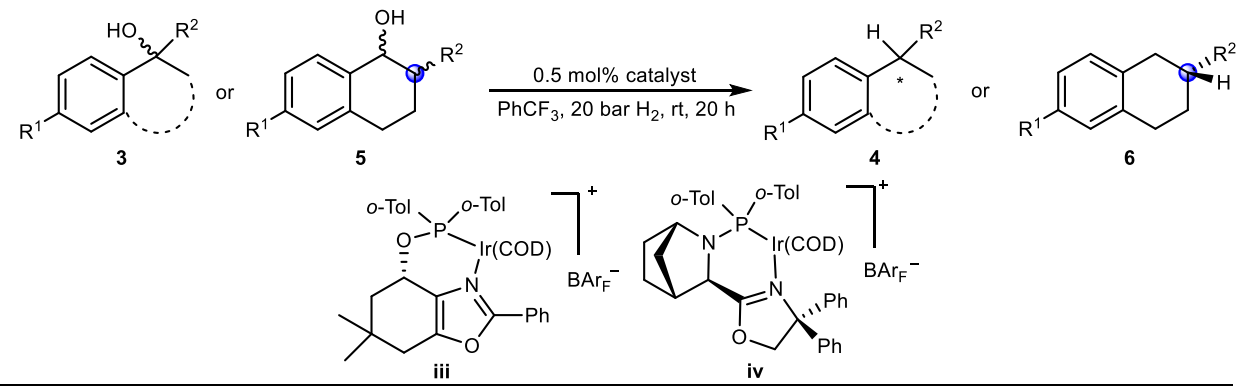

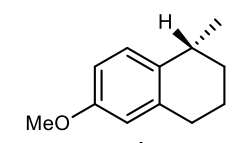

iii: $92 \%$ yield, $93 \%$ e.e.

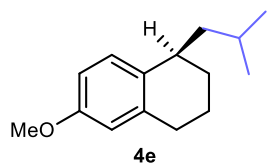

iv: $92 \%$ yield, $98 \%$ e.e.

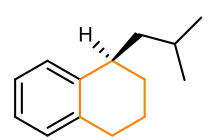

iv: $91 \%$ yield, $95 \%$ e.e

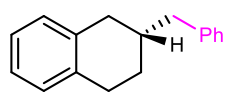

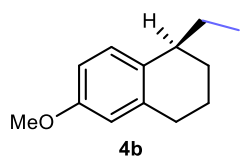

iv: $95 \%$ yield, $92 \%$ e.e.

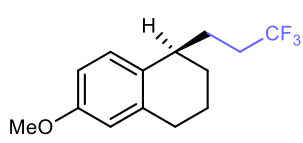

iv: $95 \%$ yield, $94 \%$ e.e.

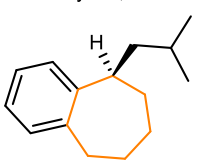

$4 \mathbf{j}$

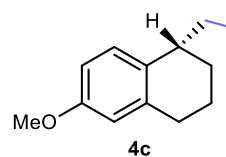

$0.88 \mathrm{~g}, 4 \mathrm{mmol}$ iii: ${ }^{\text {a }} 95 \%$ yield, $93 \%$ e.e.

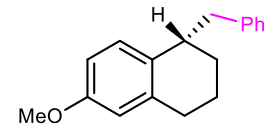

iii: $92 \%$ yield, $90 \%$ e.e.

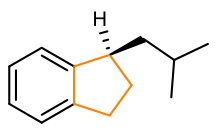

$4 \mathbf{k}$

iv: $91 \%$ yield, $98 \%$ e.e.

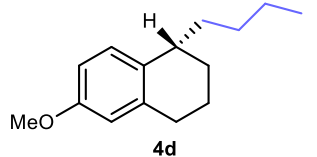

iii: $96 \%$ yield, $92 \%$ e.e.

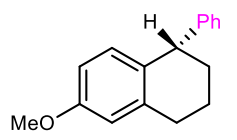

iii: ${ }^{9} 93 \%$ yield, $99 \%$ e.e.<smiles>C[C@H]1CCc2ccccc2C1</smiles>

$6 \mathbf{a}$

iv: $92 \%$ yield, $96 \%$ e.e.

iv: $96 \%$ yield, $96 \%$ e.e.

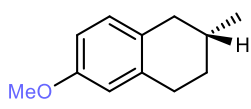

6c

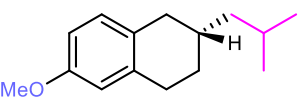

6d

iv: $97 \%$ yield, $99 \%$ e.e.

iv: $96 \%$ yield, $99 \%$ e.e.
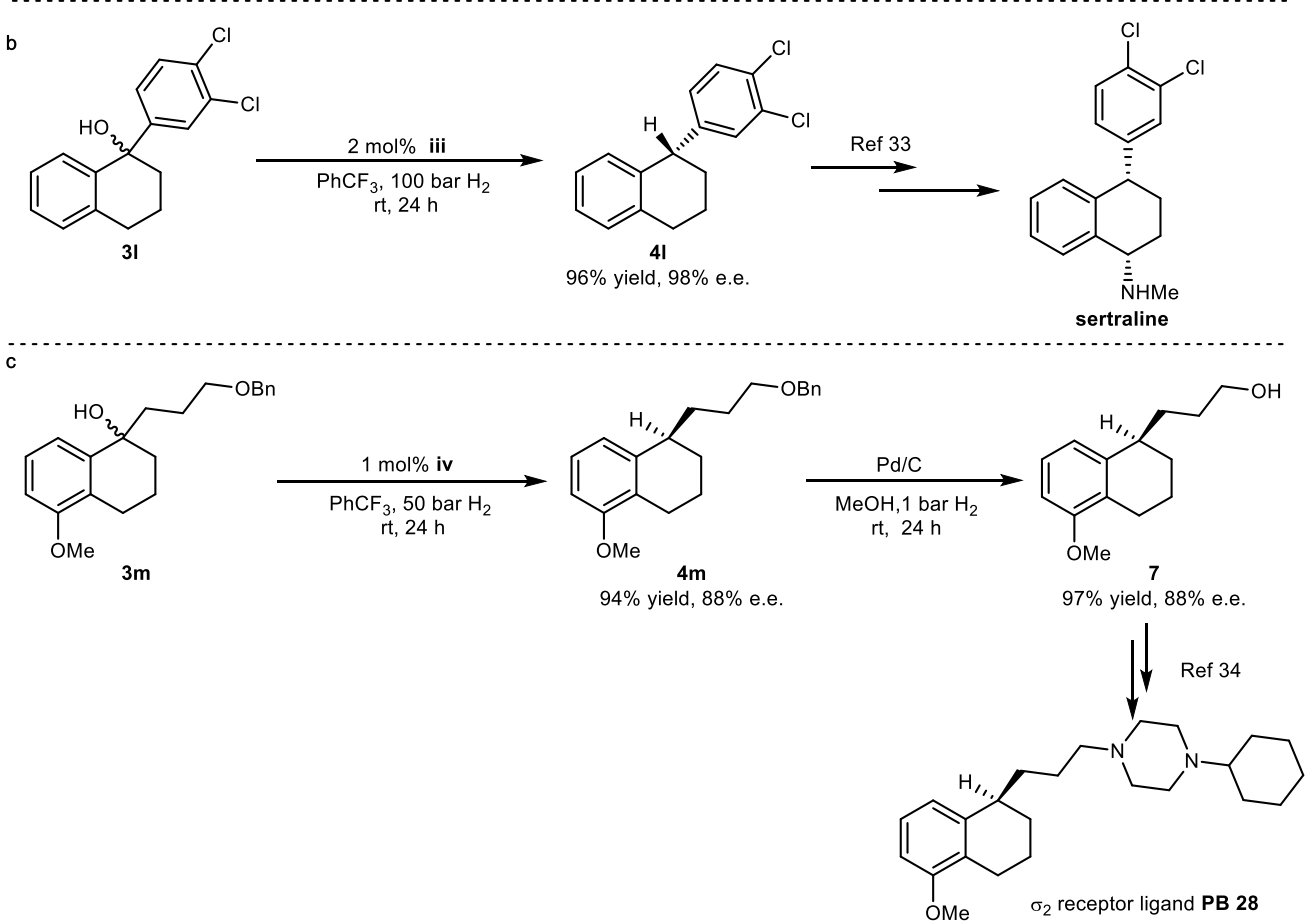

Fig. 4. Substrate scope of cyclic tertiary alcohols and synthetic applications. a, Substrate scope of cyclic

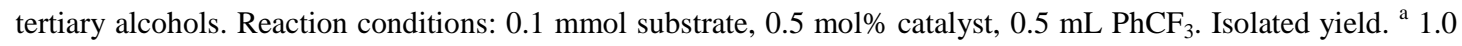
mol\% catalyst. ${ }^{b}$ DCM as solvent. Enantiomeric excess was determined by GC and SFC analysis using a chiral stationary phase, see Supplementary Table 5 for details. b, Synthesis of a sertraline intermediate. c, Synthesis of a $\sigma_{2}$ receptor intermediate. 
Mechanistic studies. As mentioned above, alcohol $1 \mathbf{k}$ provided chiral alkane $2 \mathbf{k}$ with $86 \%$ e.e., and this substrate was chosen for further studies of the mechanism of the reaction (Fig. 5a). To understand how the catalysts influenced elimination of the hydroxyl group, $\mathbf{1 k}$ was employed with the preactivated catalyst under $\mathrm{N}_{2}$ atmosphere, which led to an isomeric mixture of olefins in $16 \%$ conversion (Fig. 5b). The ratio between E-olefin 8a, terminal olefin $\mathbf{8 b}$ and Z-olefin 8c was 54.3/44.2/1.5 (Supplementary Figure 1), which suggests that the deoxygenation went through the hydrogenation of the olefin mixture. However, hydrogenation of such a mixture should result in a much lower e.e. since terminal olefins generally gives low enantioselectivity. ${ }^{3}$ To confirm this, olefin $\mathbf{8 a}$ and $\mathbf{8 b}$ were evaluated by using catalyst ii and was found to give $97 \%$ and $30 \%$ e.e., respectively (Fig. 5c). Subsequently, several labeling experiments were conducted. First, the reaction was run using deuterium gas. In this case two deuterated products were identified, 9a was obtained by hydrogenation the internal olefin (mainly $E$-olefin) while $\mathbf{9 b}$ came from the terminal olefin, which gave around 45/55 ratio (Fig. 5d, Supplementary Figure 2). The ratio of the olefin formation could be controlled by deuterated substrates and a KIE of the elimination that was found to prefer removal of the hydrogen over deuterium. Using $\mathbf{1} \mathbf{k}-d_{3}$ this effect led to increased internal olefin formation (internal/terminal $=$ $83 / 17$, Fig. 5e, Supplementary Figure 3). For substrate $1 \mathbf{k}-d_{2}$ the benzylic position was deuterated and resulted in a increased formation of the terminal olefin (internal/terminal $=15 / 85$, Fig. 5f, Supplementary Figure 4). Very surprisingly, when different olefin mixtures were formed as intermediates in the application of the methodology to substrates $1 \mathbf{k}-d_{2}, \mathbf{1 k}$ and $\mathbf{1 k}-d_{3}$, the enantiomeric purity of the product $\mathbf{2 k}$ varied only slightly ( $83 \%, 86 \%$ and $90 \%$ e.e.) (Fig. $5 \mathrm{~d}-\mathrm{f}$ ). For comparison, olefin mixtures having the same ratios were evaluated in the hydrogenations. As expected, the enantiomeric purity of the product dropped dramatically (from $88 \%$ to $37 \%$ e.e.) as the amount of terminal olefin in the mixtures increased from $17 \%$ to $83 \%$ (Fig. $5 \mathrm{~d}-\mathrm{f}$ ). The above results suggests that the enantioselectivity is not primarily dependent on the ratio of the isomeric mixture in the olefination pathway but instead mainly reliant on the interaction between the chiral iridium catalyst which in the elimination might provide olefin complexes in which predominantly one of the enantiometric faces are associated to the Ir-center. 

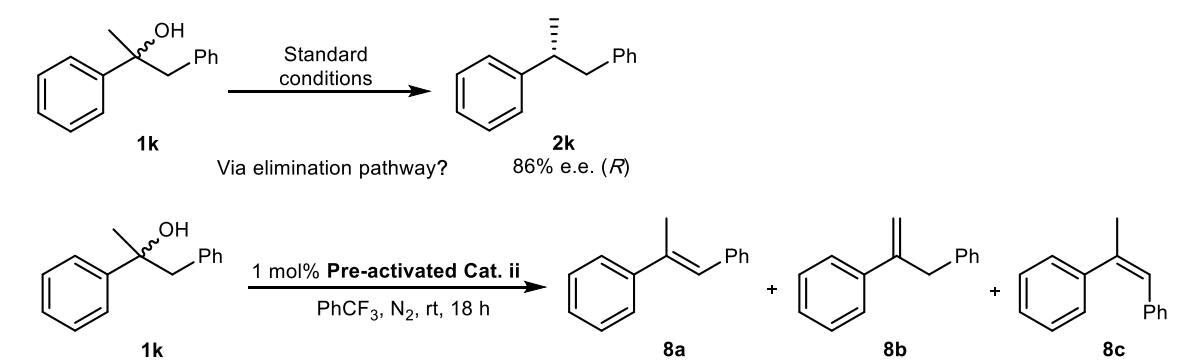

$1 k$

8 a

$8 \mathbf{8 b}$
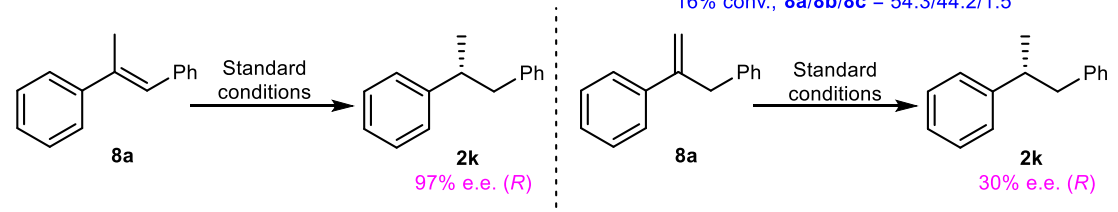<smiles>CC(O)(Cc1ccccc1)c1ccccc1</smiles>

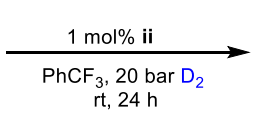<smiles>[2H]C(c1ccccc1)C(C)(c1ccccc1)c1ccccc1</smiles><smiles>[2H][C@@](O)(Cc1ccccc1)c1ccccc1</smiles>

$9 b$

Hydrogenation of same ratio olefin mixtures, $62 \%$ e.e.<smiles>CC(O)(Cc1ccccc1)c1ccccc1</smiles>

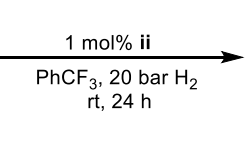<smiles>O=S(=O)(c1ccccc1)[C@H](Cc1ccccc1)c1ccccc1</smiles>

$9 c$<smiles>O=C(O)[C@H](Cc1ccccc1)c1ccccc1</smiles>

$\mathbf{9 c} / \mathbf{9 d}=83 / 17,90 \%$ e.e.

Hydrogenation of same ratio olefin mixtures, $88 \%$ e.e.
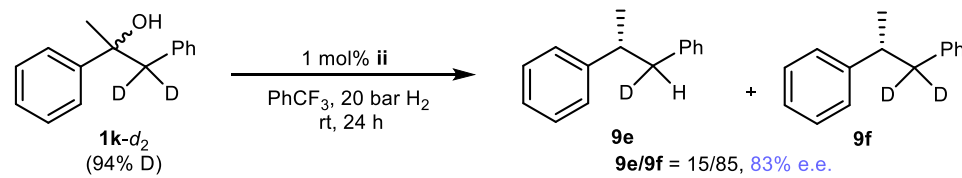

$9 \mathbf{e} / \mathbf{9 f}=15 / 85,83 \%$ e.e.

Hydrogenation of same ratio olefin mixtures, $37 \%$ e.e.

Fig. 5. Mechanistic studies. a, Enantioselective deoxygenation of $1 \mathbf{k}$ under standard conditions. b, Alcohol elimination by pre-activated catalyst. c, Asymmetric hydrogenations of geometrically pure alkenes. d, Enantioselective deoxygenation of $\mathbf{1 k}$ under $\mathrm{D}_{2}$ gas. e, Enantioselective deoxygenation of deuterated substrate $\mathbf{1 k}$ $d_{3}$. f, Enantioselective deoxygenation of deuterated substrate $1 \mathbf{k}-d_{2}$.

Computational studies. A reaction pathway involving protonation of the alcohol at the iridium catalyst was identified by density functional theory (DFT) using the B3LYP-D3 functional with implicit solvation corrections (Fig. 6, See Supplementary Methods for details of the computations). The initial structure was complex A where $\mathrm{H}_{2}$ has added oxidatively and one $\mathrm{H}_{2}$ coordinates trans to the phosphorous, while the alcohol coordinates to the metal via the hydroxyl oxygen trans to one of the hydrides. The structure is similar to the proposed structures for olefin hydrogenation ${ }^{35}$ but in the current systems olefins are only intermediates and present in low concentration. After rearrangement to coordinate via one of the phenyl groups, the hydroxyl group can accept a proton from the coordinated $\mathrm{H}_{2}$. A transition state was identified for the process at $\Delta \mathrm{G}=21.8 \mathrm{kcal} \mathrm{mol}^{-1}$ relative to $\mathbf{c 1}$. The product complex was found to be more stable on the potential energy surface, but after thermochemical corrections the Gibbs free energy was actually slightly higher than the preceding transition state. This is an artefact that mainly originates from the separation of nuclear and electronic degrees of freedom, with a posteriori correction for nuclear vibrational zero-point energy. On the potential energy surface c3ts is higher in energy than $\mathbf{c 4}$. After the oxonium complex $\mathbf{c} 4$ is formed the $\mathrm{C}-\mathrm{O}$ bond is broken via c5ts, which was found to be the highest free energy structure at $\Delta \mathrm{G}=26.6 \mathrm{kcal} \mathrm{mol}^{-1}$, which agrees well with the experimental observation of a slow but observable reaction. Once the $\mathrm{C}-\mathrm{O}$ bond is broken the carbocation initially coordinates via one of the double bonds of the phenyl ring (c6 and $\mathbf{c 7}$ ). c7 can rearrange to an $\operatorname{Ir}(\mathrm{V})$ complex $\mathbf{c 1 0}$ where the benzylic carbon forms a bond to iridium. We note 
that there is some interaction with the ipso carbon of the phenyl ring. An alternative path is direct proton transfer from the carbocation to the hydride trans to phosphorous (c8ts), but the activation energy is significant compared to that of the rearrangement. $\mathbf{c 1 0}$ is at $8.7 \mathrm{kcal} \mathrm{mol}^{-1}$ relative to $\mathbf{c 1}$, and from here the olefin can be formed via $\beta$-hydride elimination. Prior to the actual $\beta$-hydride elimination step (c13ts), two hydrides form an $\mathrm{H}_{2}$ ligand via reductive elimination (c11ts), which opens up a free coordination site at the metal. Finally, an olefin complex is formed (c14), and the process is calculated to be associated with very low activation free energies, indicating that once the $\operatorname{Ir}(\mathrm{V})$ complex $\mathbf{c 1 0}$ is formed the formation of the olefin is rapid. We have previously shown that the most likely olefin precursor for olefin hydrogenation is a complex where the olefin coordinates trans to the phosphine. We have therefore identified a mechanism by which the olefin complex can indeed rearrange via $\mathrm{H}_{2}$ dissociation and reassociation to the more stable complex (Supplementary Fig. 5). All activation energies towards the most stable complex $\mathbf{c 2 6}$ are low, with a highest point at $13.9 \mathrm{kcal} \mathrm{mol}^{-1}$.
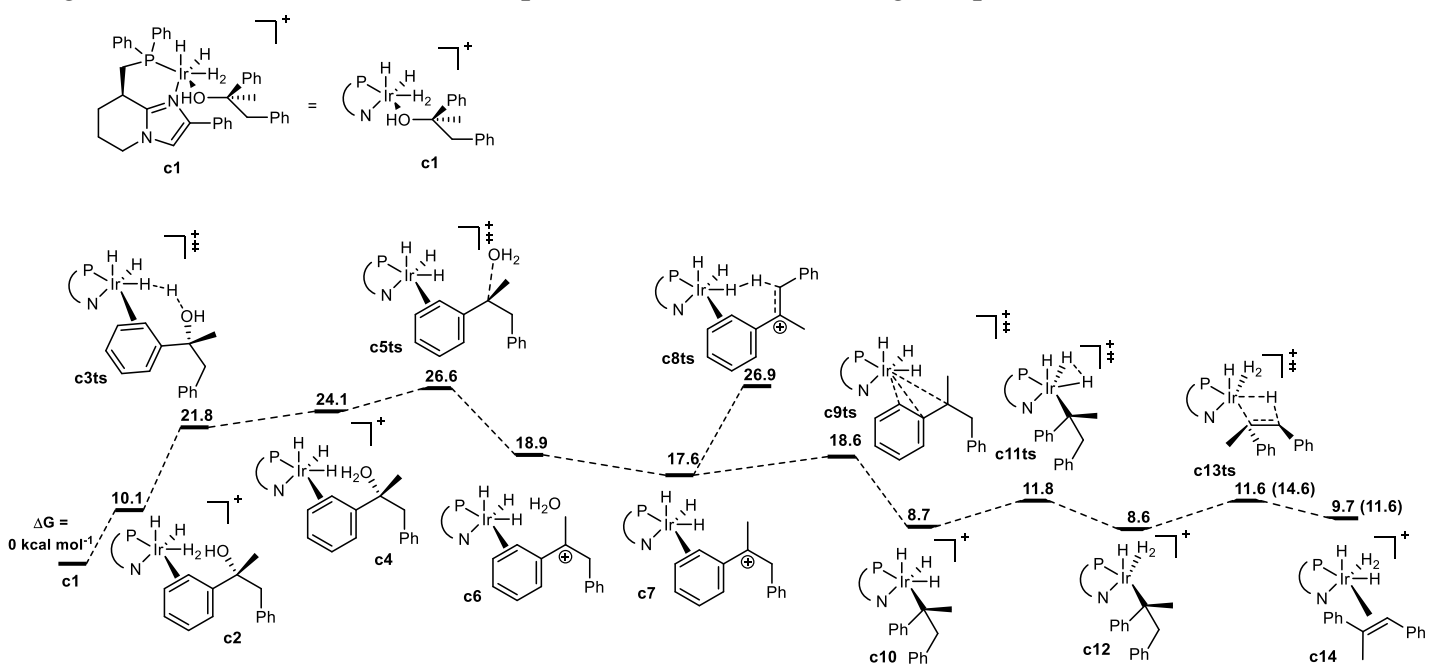

Fig. 6. Computational studies. c1-c6: Protonation of the alcohol by the $\mathrm{H}_{2}$ ligand of the iridium complex, followed by $\mathrm{C}-\mathrm{O}$ bond cleavage. c7-c14: Mechanism for formation of an olefin complex. All numbers are Gibbs free energies in $\mathrm{kcal} \mathrm{mol}^{-1}$. Numbers in parentheses are for elimination of the terminal hydride at the methyl group.

\section{Conclusions}

We have conceived and developed an N,P-iridium catalysed enantioselective deoxygenation of racemic alcohols to produce chiral alkanes. The Ir-hydride intermediates played indispensable roles in alcohol activation as well as elimination and in-situ asymmetric hydrogenation, which lead to formation of an enantioenriched tertiary carbon in the deoxygenation pathway. A variety of tertiary acyclic and cyclic alcohols and secondary cyclic alcohols have been successfully evaluated under mild conditions, giving high yields with good enantiocontrol (up to $99 \%$ e.e.). In contrast to olefins, the synthesis of alcohols does not require extensive purification and minimizes the formation of byproducts. A collection of control experiments and calculations revealed that the geometry of olefin formation is not significant for high enantioselectivity but instead suggests that the enantioselectivity originates from the iridium catalysed elimination. The presented catalytic transformation expands the possibilities of simplifying the catalytic asymmetric hydrogenation protocol and revealing an iridium mechanism in the elimination of benzylic alcohols.

\section{Methods}

General procedure for the deoxygenation of alcohols. A vial was charged with alcohol ( $0.1 \mathrm{mmol})$ and Ir-N,P-catalyst $(0.5$ or $1.0 \mathrm{~mol} \%) . \mathrm{PhCF}_{3}(0.5 \mathrm{~mL})$ was added and the vial was placed in a highpressure hydrogenation apparatus. The reactor was purged three times with Ar, and then filled with $\mathrm{H}_{2}$. The reaction was stirred at room temperature for specific time before the $\mathrm{H}_{2}$ pressure was released and the solvent removed under reduced pressure. The residue was purified by flash chromatography on 
silica gel to give the alkane. The e.e. value was determined by GC analysis or SFC analysis on chiral stationary phase. The corresponding racemic product was used for comparison and it was prepared on a $0.1 \mathrm{mmol}$ scale using $\mathrm{Pd} / \mathrm{C}$ (or racemic Ir-catalyst) as catalyst, following the same asymmetric hydrogenation procedure. See the Supplementary Methods for the characterization data, NMR spectra, GC and supercritical fluid chromatography.

\section{Data availability}

All data is available from the authors upon reasonable request.

\section{References}

1. Roseblade, S. J. \& Pfaltz, A. Iridium-catalyzed asymmetric hydrogenation of olefins. Acc. Chem. Res. 40, 1402-1411 (2007).

2. Chirik, P. J. Iron- and cobalt-catalyzed alkene hydrogenation: catalysis with both redox-active and strong field ligands. Acc. Chem. Res. 48, 1687-1695 (2015).

3. Verendel, J. J., Pàmies, O., Diéguez, M. \& Andersson, P. G. Asymmetric hydrogenation of olefins using chiral crabtree-type catalysts: scope and limitations. Chem. Rev. 114, 2130-2169 (2014).

4. Zhu, S. -F., \& Zhou, Q.-L. Iridium-catalyzed asymmetric hydrogenation of unsaturated carboxylic acids. Acc. Chem. Res. 50, 988-1001 (2017).

5. Minnaard, A. J., Feringa, B. L., Lefort, L. \& de Vries, J. G. Asymmetric hydrogenation using monodentate phosphoramidite ligands. Acc. Chem. Res. 40, 1267-1277 (2007).

6. Zhang, W., Chi, Y. \& Zhang, X. Developing chiral ligands for asymmetric hydrogenation. Acc. Chem. Res. 40, 1278-1290 (2007).

7. Etayoa, P. \& Vidal-Ferran, A. Rhodium-catalysed asymmetric hydrogenation as a valuable synthetic tool for the preparation of chiral drugs. Chem. Soc. Rev. 42, 728-754 (2013).

8. Powell, M. T., Hou, D.-R., Perry, M. C., Cui, X. \& Burgess, K. Chiral imidazolylidine ligands for asymmetric hydrogenation of aryl alkenes. J. Am. Chem. Soc. 123, 8878-8879 (2001).

9. Wang, A., Wstenberg, B. \& Pfaltz, A. Enantio- and diastereoselective hydrogenation of farnesol and $O$-protected derivatives: stereocontrol by changing the $\mathrm{C}=\mathrm{C}$ bond configuration. Angew. Chem. Int. Ed. 47, 2298-2300 (2008).

10. Peters, B. K., Zhou, T., Rujirawanich, J., Cadu, A., Singh, T., Rabten, W., Kerdphon, S. \& Andersson, P. G. An enantioselective approach to the preparation of chiral sulfones by Ir-catalyzed asymmetric hydrogenation. $J$. Am. Chem. Soc. 136, 16557-16562 (2014).

11. Liu, G., Han, Z., Dong, X.-Q. \& Zhang, X. Rh-catalyzed asymmetric hydrogenation of $\beta$-substituted- $\beta$-thio- $\alpha, \beta-$ unsaturated esters: expeditious access to chiral organic sulfides. Org. Lett. 20, 5636-5639. (2018)

12. Yan, Q., Xiao, G., Wang, Y., Zi, G., Zhang,Z. \& Hou, G. Highly efficient enantioselective synthesis of chiral sulfones by Rh-catalyzed asymmetric hydrogenation. J. Am. Chem. Soc. 141, 4, 1749-1756 (2019)

13. Takaya, H., Ohta, T., Sayo, N., Kumobayashi, H., Akutagawa, S., Inoue, S., Kasahara, I. \& Noyori, R. Enantioselective Hydrogenation of Allylic and Homoallylic Alcohols. J. Am. Chem. Soc. 109, 1596-1597 (1987).

14. Maryanoff, B. E. \& Reitz, A. B. The Wittig olefination reaction and modifications involving phosphoryl-stabilized carbanions. stereochemistry, mechanism, and selected synthetic Aspects. Chem. Rev. 89, 863-927 (1989).

15. Thiemann, T. Wittig- and Horner-Wadsworth-Emmons olefination in aqueous media with and without phase transfer catalysis. Mini-Rev. Org. Chem. 15, 412 -432 (2018).

16. Kita, Y., Hida, S., Higashihara, K., Jena, H. S., Higashida, K. \& Mashima, K. Chloride-bridged dinuclear rhodium (III) complexes bearing chiral diphosphine ligands: catalyst precursors for asymmetric hydrogenation of simple olefins. Angew. Chem. Int. Ed. 55, 8299-8303 (2016).

17. Barton, D. H. R. \& McCombie, S. W. A new method for the deoxygenation of secondary alcohols. J. Chem. Soc., Perkin Trans. 1 16, 1574-1585. (1975).

18. Nozaki, K., Oshima, K. \& Utimoto, K. Facile reduction of dithiocarbonates with $n$-Bu $\mathrm{Bu}_{3} \mathrm{Sn}_{-} \mathrm{Et}_{3} \mathrm{~B}$. Easy access to hydrocarbons from secondary alcohols. Tetrahedron Lett. 29, 6125-6126. (1988). 

Soc. 119, 6949-6950 (1997).

20. Drosos, N. \& Morandi, B. Boron-catalyzed regioselective deoxygenation of terminal 1,2-diols to 2-alkanols enabled by the strategic formation of a cyclic siloxane intermediate. Angew. Chem. Int. Ed. 54, 8814-8818 (2015).

21. Foskey, T. J. A., Heinekey, D. M. \& Goldberg, K. I. Partial deoxygenation of 1,2-propanediol catalyzed by iridium pincer complexes. ACS Catal. 2, 1285-1289 (2012).

22. Lao, D. B., Owens, A. C. E., Heinekey, D. M. \& Goldberg, K. I. Partial deoxygenation of glycerol catalyzed by iridium pincer complexes. ACS Catal. 3, 2391-2396 (2013).

23. Schlaf, M., Ghosh, P., Fagan, P. J., Hauptman, E. \& Bullock, R. M. Metal-catalyzed selective deoxygenation of diols to alcohols. Angew. Chem. Int. Ed. 40, 3887-3890 (2001).

24. Schlaf, M., Ghosh, P., Fagan, P. J., Hauptman, E. \& Bullock, R. M. Catalytic deoxygenation of 1,2-propanediol to give n-propanol. Adv. Synth. Catal. 351, 789-800 (2009).

25. Dai, X.-J. \& Li, C.-J. En route to a practical primary alcohol deoxygenation. J. Am. Chem. Soc. 138, 5433-5440 (2016).

26. Huang, J.-L., Dai, X.-J. \& Li, C.-J. Iridium-Catalyzed Direct Dehydroxylation of Alcohols. Eur. J. Org. Chem. 64966500 (2013).

27. Bauer, J. O., Chakraborty, S. \& Milstein, D. Manganese-catalyzed direct deoxygenation of primary alcohols. ACS Catal. 7, 4462-4466 (2017).

28. Yang, S., Tang, W., Yang, Z. \& Xu, J. Iridium-catalyzed highly efficient and site-selective deoxygenation of alcohols. ACS Catal. 8, 9320-9326 (2018). Isomura, M., Petrone, D. A. \& Carreira, E. M. Coordination-induced stereocontrol over carbocations: asymmetric reductive deoxygenation of racemic tertiary alcohols. J. Am. Chem. Soc. 141, 11, 4738-4748. (2019). Liu, J., Krajangsri, S., Yang, J., Li, J.-Q. \& Andersson, P. G. Iridium-catalysed asymmetric hydrogenation of allylic alcohols via dynamic kinetic resolution. Nat. Catal. 1, 438-443 (2018).

31. Krajangsri, S., Wu, H., Liu, J., Rabten, W., Singh, T. \& Andersson, P. G. Tandem Peterson olefination and chemoselective asymmetric hydrogenation of $\beta$-hydroxy silanes. Chem. Sci. 10, 3649-3653 (2019). Kerdphon, S., Ponra, S., Yang, J., Wu, H., Eriksson, L. \& Andersson, P. G. Diastereo- and enantioselective synthesis of structurally diverse succinate, butyrolactone, and trifluoromethyl derivatives by iridium-catalyzed hydrogenation of tetrasubstituted olefins. ACS Catal. 9, 6169-6176 (2019).

Poremba, K. E., Kadunce, N. T., Suzuki, N., Cherney, A. H. \& Reisman, S. E. Nickel-catalyzed asymmetric reductive cross-coupling to access 1,1-diarylalkanes. J. Am. Chem. Soc. 139, 5684-5687 (2017).

Berardi, F., Ferorelli, S., Abate, C., Colabufo, N. A., Contino, M., Perrone, R. \& Tortorella, V. 4-(Tetralin-1-yl)- and 4-(naphthalen-1-yl)alkyl derivatives of 1-cyclohexylpiperazine as $\sigma$ receptor ligands with agonist $\sigma_{2}$ activity. J. Med. Chem. 47, 2308-2317 (2004).

Church, T. L.; Rasmussen, T. \& Andersson, P. G. Enantioselectivity in the iridium catalyzed hydrogenation of unfunctionalized olefins. Organometallics, 29, 6769-6781 (2010).

\section{Acknowledgements}

The Swedish Research Council (VR), Stiftelsen Olle Engkvist Byggmästare, the Carl Tryggers Stiftelse, Knut and Alice Wallenberg foundation (KAW 2016.0072 and KAW 2018:0066) supported this work. J.J. thanks the Scientist Development Project Commemorating His Majesty the King's $84^{\text {th }}$ Birthday Anniversary. We are also thankful to Prof. David A. Tanner and Mr. Mark Nolan for proofreading the manuscript.

\section{Author contributions}

P.G.A. conceived and designed the experiments. J.Z., J.J., B.B.C.P., J.G. and S.P. performed experiments and prepared the Supplementary Information. M.S.G.A. performed the computational studies. P.G.A. and J.Z. wrote the paper. All authors discussed the results and commented on the manuscript.

\section{Present Address}


${ }^{\ddagger}$ Program in Chemical Biology, Chulabhorn Graduate Institute, Chulabhorn Royal Academy, 54 Kamphaeng Phet 6 Road, Laksi, Bangkok 10210, Thailand.

\section{Competing interests}

The authors declare no competing interests. 